\title{
Transcriptional Profiling of Immune and Inflammatory Responses in the Context of SARS-CoV-2 Fungal Superinfection in a Human Airway Epithelial Model
}

\author{
Claire Nicolas de Lamballerie ${ }^{1}$ (D), Andrés Pizzorno ${ }^{1}\left(\mathbb{D}\right.$, Julien Fouret ${ }^{2}$, Lea Szpiro ${ }^{1}$, \\ Blandine Padey ${ }^{1,2} \mathbb{D}^{\mathbb{D}}$, Julia Dubois ${ }^{1}(\mathbb{D})$, Thomas Julien ${ }^{1,3}$, Aurélien Traversier ${ }^{1}$, \\ Victoria Dulière ${ }^{1,3}$, Pauline Brun ${ }^{1,3}$, Bruno Lina ${ }^{1,4}$, Manuel Rosa-Calatrava ${ }^{1,3}$ and \\ Olivier Terrier ${ }^{1, *(D)}$ \\ 1 CIRI, Centre International de Recherche en Infectiologie, (Team VirPath), Univ Lyon, Inserm, U1111, \\ Université Claude Bernard Lyon 1, CNRS, UMR5308, ENS de Lyon, F-69007 Lyon, France; \\ claire.nicolas-de-lamballerie@univ-lyon1.fr (C.N.d.L.); mario-andres.pizzorno@univ-lyon1.fr (A.P.); \\ 1.szpiro@virhealth.fr (L.S.); Blandine.padey@univ-lyon1.fr (B.P.); Julia.dubois@univ-lyon1.fr (J.D.); \\ thomas.julien@univ-lyon1.fr (T.J.); aurelien.traversier@univ-lyon1.fr (A.T.); \\ victoria.duliere@univ-lyon1.fr (V.D.); pauline.brun15@orange.fr (P.B.); bruno.lina@univ-lyon1.fr (B.L.); \\ manuel.rosa-calatrava@univ-lyon1.fr (M.R.-C.) \\ 2 Signia Therapeutics SAS, F-69008 Lyon, France; julien.fouret@signiatherapeutics.com \\ 3 VirNext, Faculté de Médecine RTH Laennec, Université Claude Bernard Lyon 1, Université de Lyon, \\ F-69008 Lyon, France \\ 4 Laboratoire de Virologie, Centre National de Référence des virus Influenza Sud, Institut des Agents \\ Infectieux, Groupement Hospitalier Nord, Hospices Civils de Lyon, F-69004 Lyon, France \\ * Correspondence: olivier.terrier@univ-lyon1.fr
}

Received: 3 November 2020; Accepted: 10 December 2020; Published: 11 December 2020

\begin{abstract}
An increasing amount of evidence indicates a relatively high prevalence of superinfections associated with coronavirus disease 2019 (COVID-19), including invasive aspergillosis, but the underlying mechanisms remain to be characterized. In the present study, to better understand the biological impact of superinfection, we determine and compare the host transcriptional response to severe acute respiratory syndrome coronavirus 2 (SARS-CoV-2) versus Aspergillus superinfection, using a model of reconstituted human airway epithelium. Our analyses reveal that both simple infection and superinfection induce strong deregulation of core components of innate immune and inflammatory responses, with a stronger response to superinfection in the bronchial epithelial model compared to its nasal counterpart. Our results also highlight unique transcriptional footprints of SARS-CoV-2 Aspergillus superinfection, such as an imbalanced type I/type III IFN, and an induction of several monocyte and neutrophil associated chemokines, that could be useful for the understanding of Aspergillus-associated COVID-19 and also the management of severe forms of aspergillosis in this specific context.
\end{abstract}

Keywords: SARS-CoV-2; Aspergillus; invasive aspergillosis; transcriptional profiling; COVID-19

\section{Introduction}

The current pandemic of novel coronavirus disease 2019 (COVID-19), caused by severe acute respiratory syndrome coronavirus 2 (SARS-CoV-2), had the first cases reported in Wuhan, Hubei province, China, in December 2019. As of 5 December 2020, there have been more than 65,007,974 confirmed COVID-19 cases in the world as reported by the WHO, including 1,507,018 deaths (WHO). 
SARS-CoV-2 is a beta-coronavirus closely related to the severe acute respiratory syndrome coronavirus (SARS-CoV) and the Middle East respiratory syndrome coronavirus (MERS-CoV) that emerged in 2003 and 2012, respectively.

In a short period of time, significant effort has been devoted to understanding the molecular basis of the pathology associated with SARS-CoV-2 infection in an attempt to guide work on treatment, vaccine, and diagnostic test development. Numerous clinical studies have reported that the pathophysiology of COVID-19 has similar aspects to that initially described for SARS-CoV, i.e., acute lung injury due to over-inflammation following early stages driven by infection and viral replication [1-5]. Nevertheless, the underlying mechanisms of these exuberant inflammatory responses in SARS-CoV-2 infection remain largely unknown, and there is a need to expand our knowledge of the host's response. In this context, several recent omics-based approaches, including in vivo and in vitro transcriptional profiling studies, have highlighted specific aspects of the signature of infection that could contribute to COVID-19 [6-9]. Blanco-Melo and colleagues, using transcriptional and serum profiling of COVID-19 patients, have notably shown that the SARS-CoV-2 infection signature was defined by low levels of Type I and III interferons juxtaposed to elevated chemokines and high expression of IL- 6 [6]. A recent report has shown that inborn errors of TLR3- and IRF7-dependent type I IFN immunity can underlie severe COVID-19 pneumonia in patients with no prior severe infection [10].

It is now well known that superinfections of bacterial/fungal origin can affect the course and severity of respiratory viral infections. For example, the co-pathogenesis of viruses and bacteria into the lung has been extensively studied, notably in the context of influenza superinfection by bacteria such as S. pneumoniae [11-14]. To date, there are limited data available on superinfections associated with COVID-19 [15], though superinfections were reported in 10\%-20\% of SARS-CoV-2-infected adults admitted to Wuhan hospitals through the end of January 2020 , and notably in $50 \%-100 \%$ of those who died [16]. In intensive care units, COVID-19 patients are at high risk of developing secondary infections, including fungal infections, e.g., invasive pulmonary aspergillosis [17]. Indeed, a recent study on the French COVID-19 cohort reported that 33\% of critically ill COVID-19 patients also showed invasive aspergillosis [18]. However, while the reasons for increased vulnerability to Aspergillus in COVID-19 patients remain undetermined, the putative contribution of Aspergillus to SARS-CoV-2 related lung inflammation and COVID-19 pathophysiology also constitutes a major unanswered question.

To better understand the biological impact of superinfection in the SARS-CoV-2 context, we sought to determine and compare the host transcriptional response to SARS-CoV-2 versus a SARS-CoV-2 + Aspergillus superinfection. To reach this goal, we established a model of SARS-CoV-2 infection and superinfection in reconstituted human airway epithelia (HAE), based on previously published works [19-21]. Our analysis reveals that both simple infection and superinfection induced strong deregulation of core components of innate immune and inflammatory responses, however, it also highlighted unique transcriptional footprints of the SARS-CoV-2 + Aspergillus superinfection that provide valuable insight for the understanding not only of Aspergillus-associated COVID-19 but also for the management of severe forms of aspergillosis.

\section{Materials and Methods}

\subsection{Reconstituted Human Airway Epithelial Model}

MucilAir ${ }^{\mathrm{TM}}$ HAE reconstituted from primary human cells obtained from nasal or bronchial biopsies, were provided by Epithelix SARL (Geneva, Switzerland) and maintained in air-liquid interphase, according to the manufacturer's instructions. For infection experiments, apical poles were gently washed twice with warm OptiMEM medium (Gibco, ThermoFisher Scientific, Waltham, MA, USA) and then infected directly with $150 \mu \mathrm{L}$ dilution of the virus in OptiMEM medium, at a multiplicity of infection (MOI) of 0.1 (i.e., $5 \times 10^{4}$ TCID50/epithelium) [21]. For mock infection, the same procedure was followed using OptiMEM as inoculum. Superinfection was performed in SARS-CoV-2 infected cells at $48 \mathrm{~h}$ post-infection, with apical inoculation of $10 \mu \mathrm{L}$ of Aspergillus in OptiMEM at a MOI of 1. 
Samples were collected from apical washes or basolateral medium at $72 \mathrm{~h}$ post-infection. Variations in transepithelial electrical resistance (TEER) were measured using a dedicated volt-ohm meter (EVOM2, Epithelial Volt/Ohm Meter for TEER) and expressed as Ohm.cm².

\subsection{Pathogens}

All experiments involving clinical samples and the manipulation of infectious SARS-CoV-2 were performed in biosafety level 3 (BSL-3) facilities, using appropriate protocols. The BetaCoV/France/ IDF0571/2020 SARS-CoV-2 strain used in this study was isolated directly from a patient sample, as described elsewhere [21]. Viral stocks were prepared and quantified in Vero E6 cells (TCID50/mL). Aspergillus niger (ATCC 16404) was maintained on potato dextrose agar for $5-7$ days at $37^{\circ} \mathrm{C}$. Conidia were then harvested by washing culture flask with sterile PBS supplemented with $0.1 \%$ Tween 20 and quantified on maltose extract agar plates (CFU/mL).

\section{3. $m R N A$ Sequencing}

Total RNA was extracted using the RNeasy Mini Kit (QIAGEN, ref. 74104, Courtaboeuf, France) with DNase treatment, following the manufacturer's instruction. $500 \mu \mathrm{g}$ total RNA was used to prepare polyA-enriched RNA-seq libraries using the KAPA mRNA Hyper Prep Kit (Roche, ref. KK8581/KK8581, Basel, Switzerland). Those libraries were prepared separately for each sample with 11 amplification cycles, and then all libraries were equimolar pooled before sequencing. Paired-end sequencing $(2 \times 100 \mathrm{bp})$ was performed with Illumina NovaSeq 6000 sequencing platform on an SP flowcell (Illumina, ref. 20040326, San Diego, CA, USA). Raw reads were first cleaned with Cutadapt 2.8 [22] to trim adapters and low-quality ends (i.e., terminal bases with Phred quality score below 30). Only reads longer than $75 \mathrm{bp}$ and with less than $20 \%$ of $\mathrm{N}$ (undetermined base) after trimming were kept for further analysis. The Kallisto 0.46.1 software was used for reference-indexing and transcript abundance estimation [23]. The reference transcriptome based on NCBI RefSeq annotation release 109.20191205 and genome assembly build GRCh37.p13 were chosen for this analysis [24]. Table S2 provides a summary statistic of the pseudo-alignment process.

\subsection{Differential Expression Analysis}

Differential expression analysis was performed with R 3.6.3. First, abundances at transcript-level were imported using tximport 1.14.0 [25]. Only coding mRNA transcripts were considered. Gene-level effective lengths were obtained by weighted mean with their expression values in FPKM (Fragment Per Kilobase Million) weights. Raw counts were also computed at gene-level by sum and used as input for DESeq2 1.26.0 [26]. Normalization within DESeq2 used first gene-level effective length, and then the size factor was estimated using the function 'estimateSizeFactors.' Differential expression testing was performed using default parameters. P-values were adjusted using the Benjamini-Hochberg method after the independent filtering was implemented by default in DESeq2. A gene was considered differentially expressed if both its adjusted p-value was below 0.01 and its induced change in expression was at least a 2-fold increase (for upregulated genes) or a 2-fold decrease (for down-regulated genes) compared to the control condition. Finally, the NCBI gene IDs were mapped to Uniprot IDs using Uniprot cross-references (https://www.uniprot.org/database/DB-0118) [24,27]. First, Uniprot entries associated with several gene IDs were removed. Indeed, we were not focused on gene fusion products or read-through transcripts in this study. The protein-protein interaction (PPI) network was analyzed with STRING 11.0 and visualized with Cytoscape 3.8.0. 


\subsection{In silico Functional Analysis}

Based on mapped Uniprot IDs, gene set enrichment was performed using the parent-child union method as previously described [28]. In this method each functional term, called child, was considered relatively to its parents to compute the probability of enrichment ( $p$-value). We considered 3 functional databases with parent-child relations: Reactome, Uniprot Keyword (restricted to "Biological process" and "Disease"), and Gene ontology (Restricted to "Biological process"). Different files (available online: URLs were all accessed the 04/02/2020) were parsed to define Uniprot ID associations with terms and identify a child to parent relations within each functional database; URLs are specified in Table S1.

\section{Results}

\subsection{Simple Infection and Superinfection Induce a Strong Deregulation of Core Components of Innate Immune} and Inflammatory Responses

In order to identify similarities and differences between the host response to SARS-CoV-2 simple infection and Aspergillus superinfection, we sought to investigate the transcriptome of human respiratory epithelial cells during infection, in comparison with non-infected cells. Aware of the inherent limitations of experimental models using cell lines, we set up a model of infection/superinfection in a physiological model of reconstituted human airway epithelium (HAE). Developed from biopsies of nasal or bronchial cells differentiated in the air/liquid interphase, these HAE models, that we previously used with different respiratory viruses including SARS-CoV-2 [19-21], reproduced with high fidelity most of the main structural, functional, and innate immune features of the human respiratory epithelium that play a central role during infection, hence constituting an interesting surrogate to study airway disease mechanisms.

We infected nasal or bronchial HAE with SARS-CoV-2, and superinfection with Aspergillus was performed at $48 \mathrm{~h}$ post-infection (hpi), which we had previously defined as the peak of acute SARS-CoV-2 infection in the HAE model [21]. Mock-infected, infected (CoV), and superinfected samples $(\mathrm{CoV}+\mathrm{Asp})$ were harvested at $72 \mathrm{hpi}(24 \mathrm{~h}$ after superinfection) to perform mRNA-seq analysis (Figure 1A). Both nasal and bronchial HAE models of superinfection were further characterized and validated in terms of viral production, impact on trans-epithelial resistance, and apical release of IL-6, which we used as hallmarks of infection (Figure 1A). Interestingly, in contrast with nasal HAE, we observed a significant increase in the relative viral production in the context of superinfection in bronchial HAE, associated with higher IL-6 levels and a stronger negative impact on trans-epithelial resistance (Figure 1B).

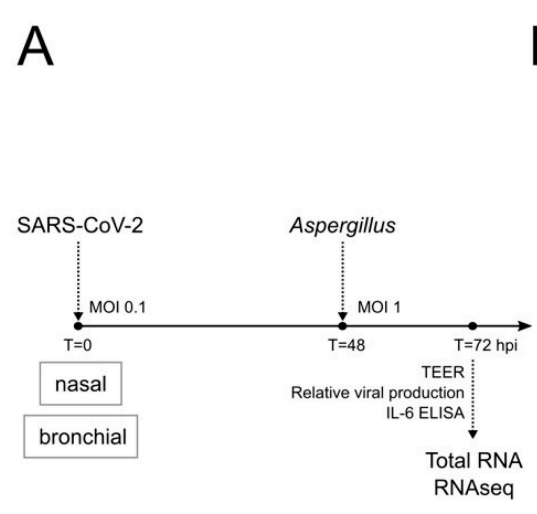

B
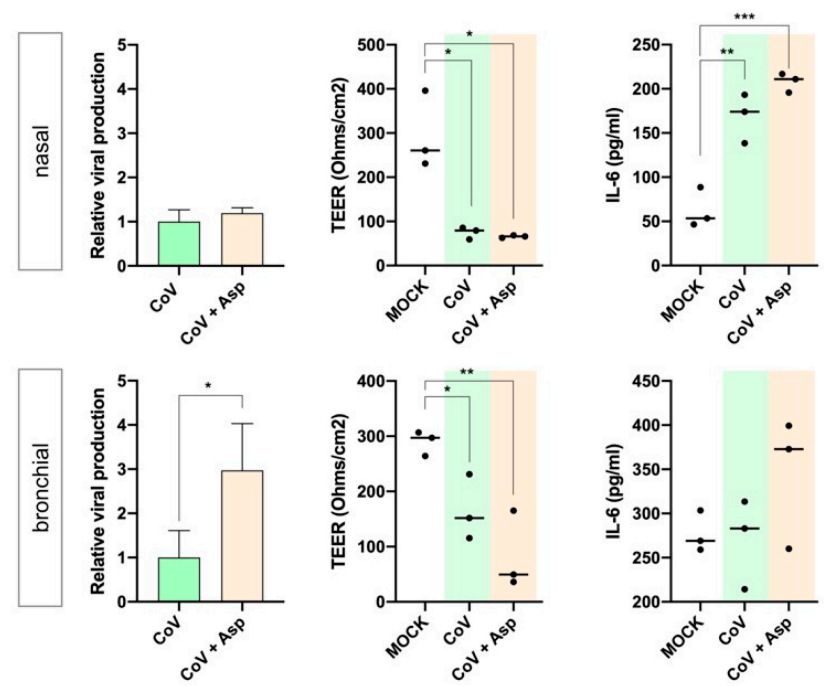

Figure 1. Cont. 

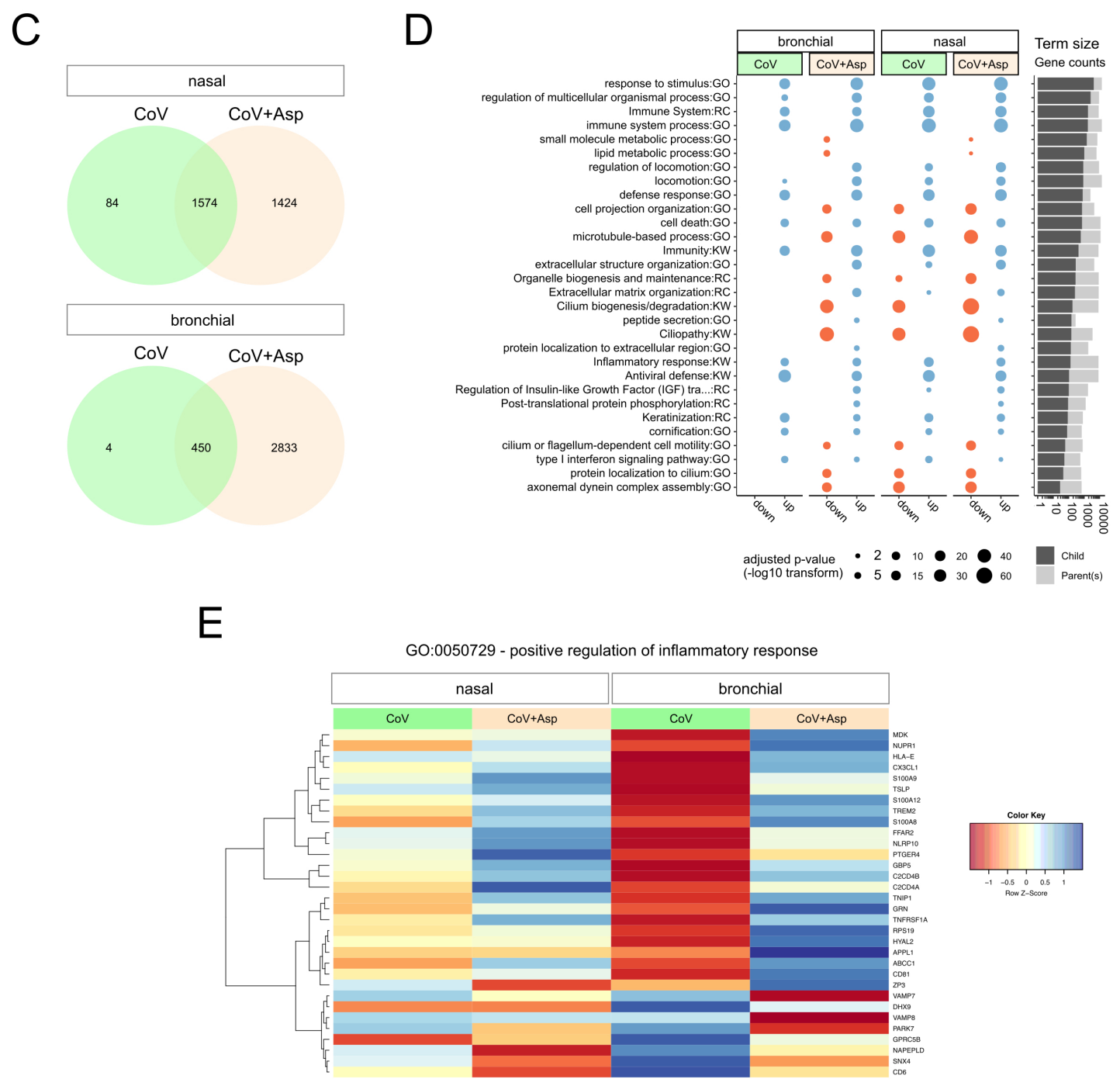

Figure 1. (A) Overview of experimental strategy. (B) At $72 \mathrm{~h}$ post-infection, for both nasal and bronchial human airway epithelia (HAE) model, the relative viral production (intracellular) was determined using RTqPCR, and the impact of infection on epithelium integrity was monitored by the measure of the transepithelial resistance (TEER Ohms $/ \mathrm{cm}^{2}$ ). IL-6 was measured at the apical using a specific ELISA assay. ${ }^{*} p<0.05 ;{ }^{* *} p<0.01 ;{ }^{* * *} p<0.001$. (C) Nasal and bronchial gene signature overlap for SARS-CoV2 or SARS-CoV-2+Aspergillus infected conditions vs. Mock. We contrasted the significantly differentially expressed gene lists corresponding to $\mathrm{CoV}$ vs. Mock and CoV+Asp vs. Mock in nasal and bronchial HAE. Only genes above threshold $(\log 2(\mathrm{FC})>1$ or $<-1$ compared to the mock-infected condition and Benjamini-Hochberg adjusted $p$-value $<0.01$ ) were considered. (D) Overview of functional enrichment results for SARS-CoV2 or SARS-CoV-2+Aspergillus infected conditions vs. Mock. Considering (CoV vs. Mock) and (CoV+Asp vs. Mock) for both bronchial and nasal HAE, significantly up- or down-regulated gene lists ( $x$-axis) were tested for significant enrichment using the parent-child strategy (see methods). If below the threshold, the adjusted p-values corresponding to different terms ( $y$-axis) are represented by point sizes (see legend). After clustering terms based on gene occurrences (binary distance and Ward algorithm) in 15 metagroups, only the top two (lowest adjusted p-value) were represented here. For the complete list of significant terms, see Figure S1. The bar plot on the right represents the sizes of enriched terms (called child) in comparison to the size of their parents (see Section 2). (E) Positive regulation of the inflammatory response in nasal and bronchial HAE. We extracted the list of proteins associated with the term GO:0050729 and visualized the scaled log-transformed expression results in a heatmap. Each row represents a gene, and each column is an experimental condition. The color key indicates the scaled expression levels vs. Mock (red, low; blue, high). 
3.2. CoV+Asp Superinfection Transcriptomic Signature Recapitulates the Signature CoV Infection, but with a Distinct Regulation of the Inflammatory Response

Differential expression analysis of mRNA-seq data compared to the mock-infected condition identified 1638 and 454 differentially expressed genes (DEGs) in SARS-CoV-2-infected nasal and bronchial HAE, respectively (FC $\geq 2$, Benjamini-Hochberg adjusted $p$-value $<0.01$ ). In the context of $\mathrm{CoV}+$ Asp superinfection, the number of DEGs was notably higher, with 2979 and 3235 genes in nasal and bronchial HAE, respectively (Supplementary Data File 1). Interestingly, there was an important overlap between $\mathrm{CoV}$ infection and CoV+Asp superinfection associated DEGs in both nasal and bronchial models. As illustrated in the Venn diagrams (Figure 1C), more than 96\% (nasal HAE) and 99\% (bronchial $\mathrm{HAE}$ ) of DEGs of the CoV signature were also part of the CoV+Asp signature. To provide a further functional interpretation of the global transcriptional signatures, we performed functional enrichment analysis on the $\mathrm{CoV}$ and $\mathrm{CoV}+\mathrm{Asp}$ nasal and bronchial models. Gene Ontology (GO), UniProt $(\mathrm{KW})$, and Reactome (RC) terms were considered enriched when their Bonferroni-adjusted corrected enrichment $p$-value was $<0.05$ (Figure 1D). As anticipated, a large part of the most enriched and shared terms between all experimental conditions was related to the host response to infection (regulation of response to cytokine stimulus, regulation of defense response, regulation of response to biotic stimulus) and also to cornification, which regroups genes mostly involved in cell-death mechanisms (Figure 1D). Interestingly, functional enrichments specific to $\mathrm{CoV}$ infection or $\mathrm{CoV}+\mathrm{Asp}$ superinfection were also highlighted. For example, gene clusters harboring terms associated with epithelium physiology and cilium movements (regulation of cellular component movements, cell projection assembly, intraflagellar transport) but also with intra- and extra-cellular signaling (post-translational protein phosphorylation, protein localization to the extracellular membrane, peptide secretion) were exclusively enriched in the context of $\mathrm{CoV}+$ Asp superinfection (Figure 1D). In parallel, given the importance of the exacerbated immune response observed mainly in severe COVID-19 cases, we further analyzed the inflammation-related terms and identified similar, yet to a different extent, regulation patterns for genes involved in the positive regulation of inflammatory response (GO:0050729) between nasal and bronchial HAE. As exemplified in the heatmap (Figure 1D), deregulated genes such as calgranulin coding genes S100A12 and S100A9/A8 and downregulated genes such as vesicle-associated membrane protein coding genes VAMP7 or VAMP8 were identified as a hallmark of CoV+Asp superinfection, in contrast to $\mathrm{CoV}$ infection (Figure 1D). Similar observations were performed using different terms related to inflammation (Figure S1), hence suggesting a very different inflammation signature resulting from superinfection. Altogether, our results indicate that the $\mathrm{CoV}+\mathrm{Asp}$ superinfection presents a transcriptomic signature that recapitulates the overall signature of a simple CoV infection, but with both a particularly distinct regulation of the inflammatory response and the additional regulation of many biological processes related to the physiology of epithelia. Of note, we observed a relatively similar global pattern of regulation between the nasal and bronchial HAE models, differing primarily in the magnitude rather than the nature of the responses to $\mathrm{CoV}$ and $\mathrm{CoV}+\mathrm{Asp}$ superinfection. 


\subsection{Transcriptional Footprints of SARS-CoV-2 Aspergillus Superinfection}

In order to explore in more depth, the transcriptomic signature of the superinfection, we then performed a differential analysis of $\mathrm{CoV}+\mathrm{Asp}$ signature using that of $\mathrm{CoV}$ infection as a baseline. This focused analysis highlighted 248 and 769 DEGs in the CoV+Asp nasal and bronchial HAE, respectively ( $\mathrm{FC} \geq 2$, adjusted $p$-value $<0.01$ ), which allowed us to spot finer differences between the two epithelium models. Indeed, the volcano plots in Figure 2A,B representing all DEGs induced by the CoV+Asp versus the $\mathrm{CoV}$ condition showed interesting differences in both the scale of deregulation and the nature of the most deregulated genes between the upper and lower respiratory tract tissues. Interestingly, many genes involved in the regulation of the inflammatory response, such as HOX1, IL1B, IL1A, and IL17C, were found amongst the most upregulated genes in the nasal HAE model (Figure 2A). These genes were also upregulated in the bronchial HAE model (Supplementary Data File 2). To provide a further functional interpretation of the superinfection signature, we performed a functional enrichment analysis, using the same strategy previously described. We only observed a limited number of enriched clusters of downregulated genes, mostly in bronchial HAE, all of them being related to epithelial physiology and cell movement (e.g., cytoskeleton-dependent intracellular transport, protein localization to the cilium, cilium movement GO terms, Figure 2C). In line with this observation, our analysis highlighted several clusters of enriched upregulated genes related to epithelial physiology (e.g., locomotion, movement of the cell, or subcellular component $\mathrm{GO}$ terms, Figure 2C). On the other hand, terms related to signaling, host response, and immunity were markedly more upregulated in the bronchial HAE model (Figure 2C and Figure S2). In addition to the gene enrichment profile shared between the nasal and bronchial models, our study also highlighted several overlapping biological processes such as the cytokine signaling and IL10 signaling pathways (Figure 2C), which is consistent with the most upregulated DEGs shown in Figure 2A and Supplementary Data File 2.

To better visualize these observations, we applied a protein-protein interactions analysis using the STRING database to investigate the DEGs corresponding to several Reactome and GO terms (immune system process, cytokine signaling in the immune system, inflammatory response, interleukin-10 signaling) enriched in the bronchial and nasal superinfection signatures along with their functional interactions (Figure 3A,B). The two interactome maps with mostly upregulated DEGs illustrate the strong functional interconnections among several cytokines/chemokines (blue) and receptors (yellow) involved in the immune and inflammatory responses occurring in the context of a SARS-CoV-2 + Aspergillus superinfection. Interestingly, our analysis underlined the role of type III IFN (IFNL1, INFL2, and INFL3) and several cytokine-coding genes such as CXCL2/CXCL8 (Figure 3A,B) that are upregulated following simple SARS-CoV-2 infection and even more upregulated in the context of superinfection, arguably illustrating an enhanced specific response to control infection in both models. 
A
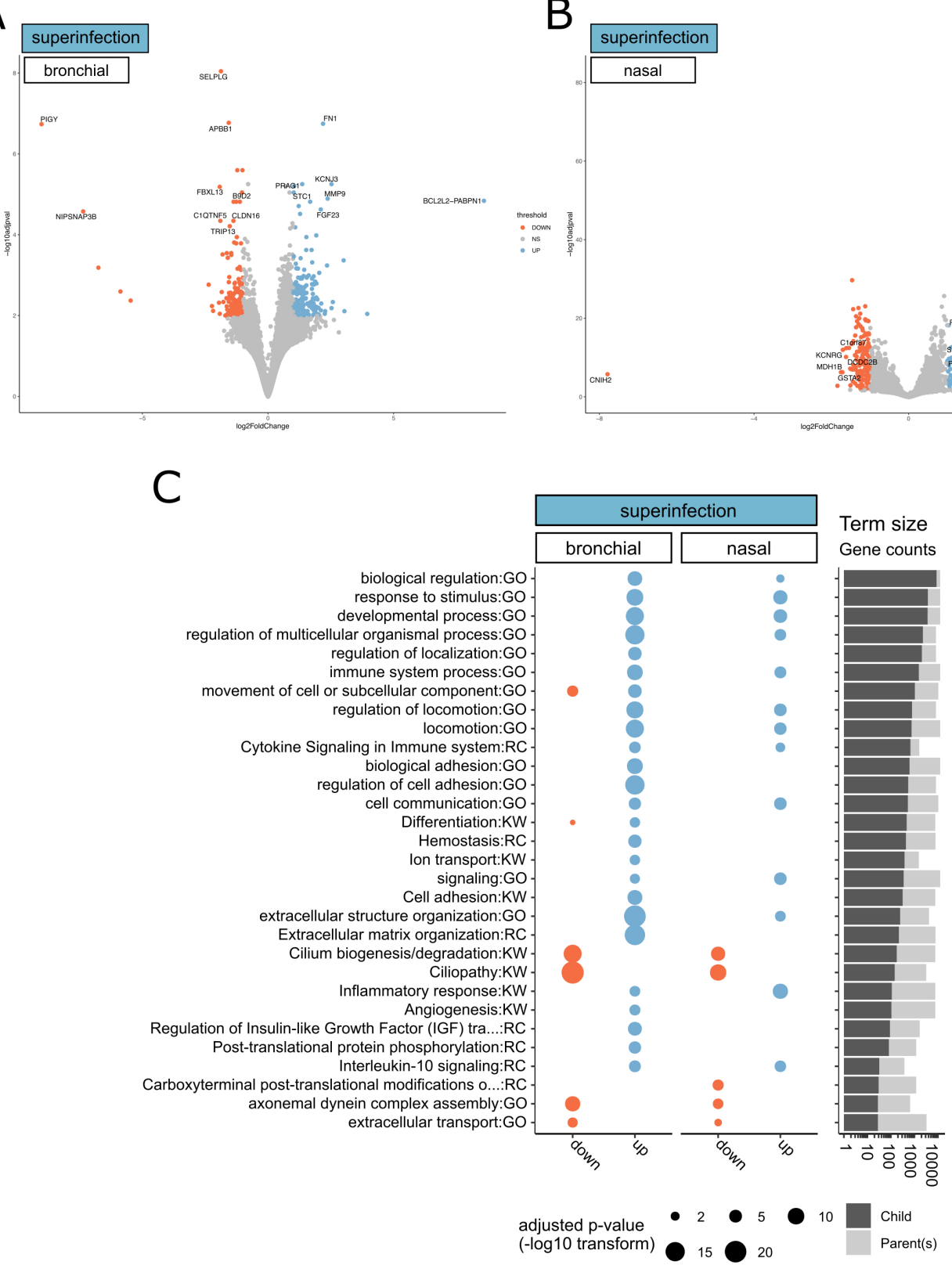
(-log10 transform)

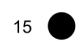

B

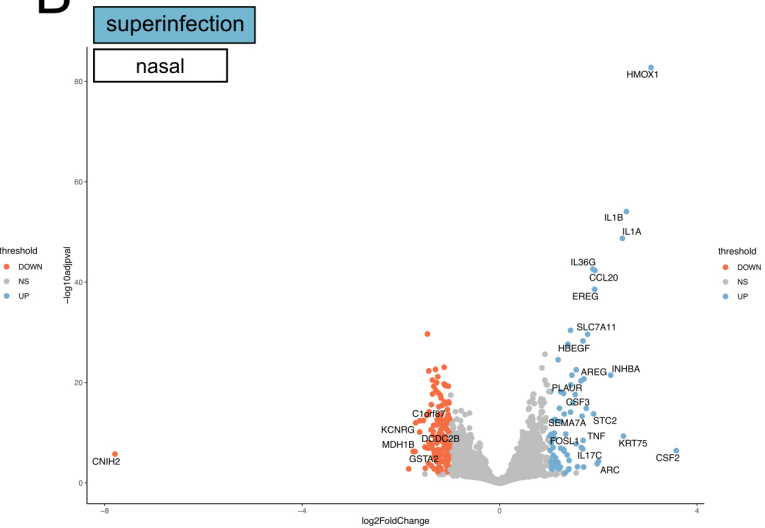

Figure 2. (A,B) Volcano plots of differentially expressed genes from (A) nasal and (B) bronchial tissue superinfected by Aspergillus. We considered significantly modulated genes (CoV+Asp vs. $\mathrm{CoV})$ for both bronchial and nasal HAE $(\log 2(\mathrm{FC})>1$ or $<-1$ compared to the $\mathrm{CoV}$ condition and Benjamini-Hochberg adjusted $p$-value $<0.01)$. The color scale represents the log-transformed fold change values and ranges from low (red) to high (blue). (C) Overview of functional enrichment results of SARS-CoV-2+Aspergillus superinfection vs. SARS-CoV-2 infection. Considering CoV+Asp vs. CoV for both bronchial and nasal epithelium type, significantly up- or down-regulated gene lists ( $x$-axis) were tested for significant enrichment using the parent-child strategy (see methods). If below the threshold (0.05), the adjusted p-values corresponding to different terms ( $y$-axis) are represented by point sizes (see legend). After clustering terms based on gene occurrences (binary distance \& Ward algorithm) in 15 metagroups, the two terms with the lowest p-value are represented here. For the complete list of significant terms, see Supplementary Data File 2. The bar plot on the right represents the sizes of enriched terms (called child) in comparison to the size of their parents (see Section 2). 
A

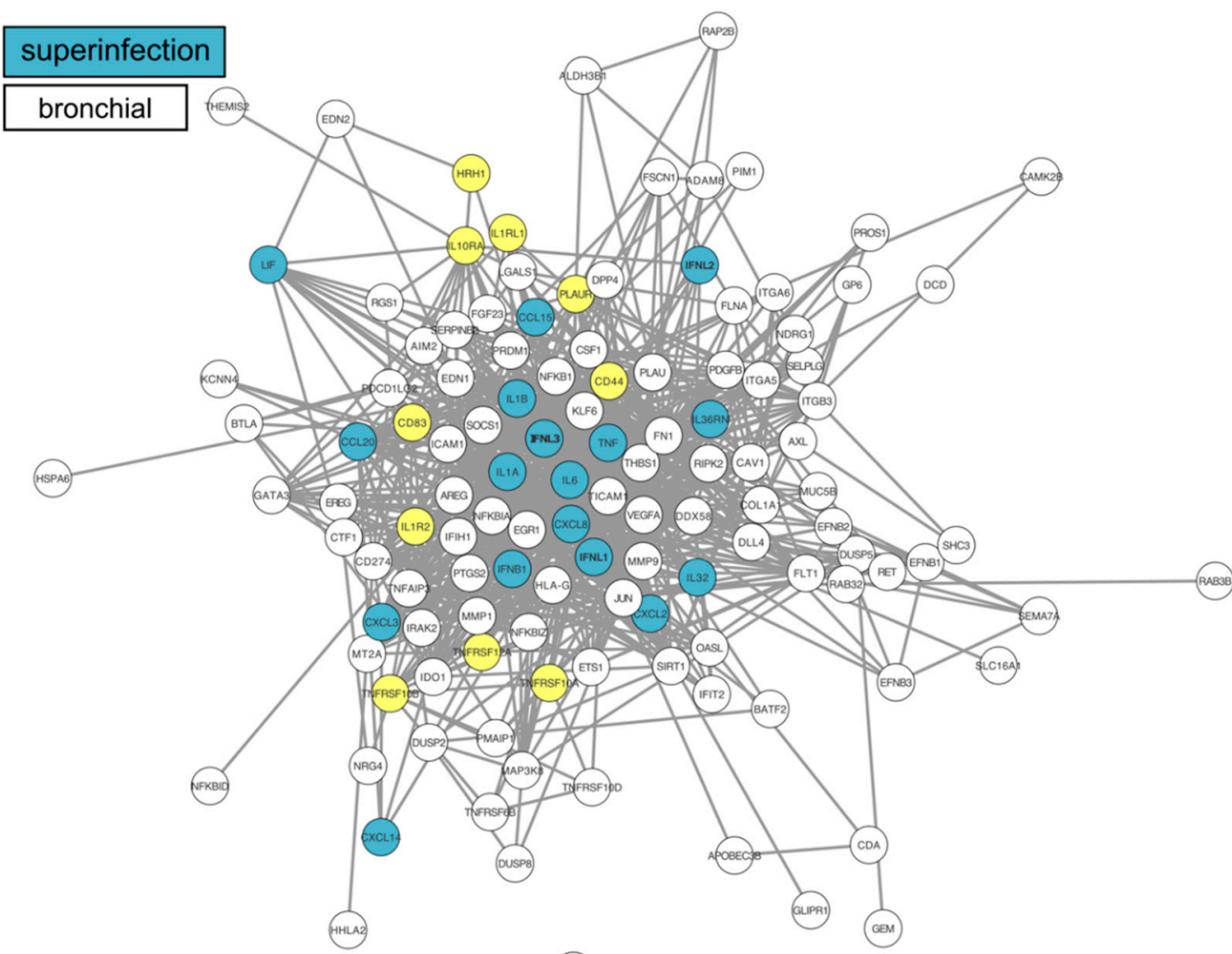

B

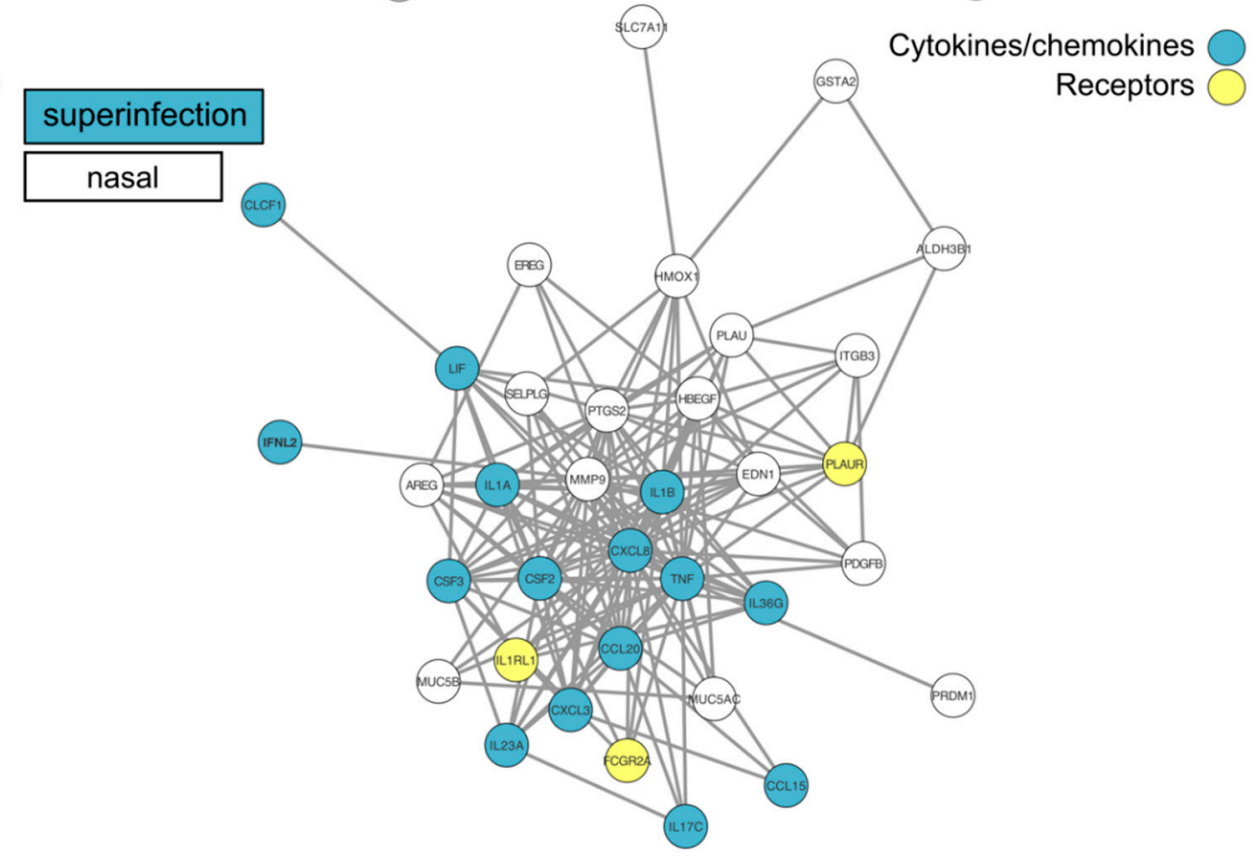

Figure 3. (A) and (B) Network visualization of immunity-associated proteins in superinfected HAE. We selected the 76 and 191 genes significantly differentially expressed (vs. CoV) in our study (respectively in nasal (A) and bronchial (B) HAE) associated with the Reactome "Interleukin-10 signaling" (R-HSA-6783783) and "cytokine signaling in immune system" pathways (R-HAS-1280215), Table 0395. and the Gene Ontology term "immune system process" (GO:0002376). The circles indicate genes modulated in our study in the superinfection context. The predicted associations are materialized by the dark lines. Cytokines/chemokines are highlighted in blue and receptors in yellow. 


\section{Discussion}

Invasive pulmonary aspergillosis (IPA), which typically occurs in an immunocompromised host, represents an important cause of morbidity and mortality worldwide [29]. Superinfections were extensively documented in the case of influenza infections, with the latter being usually described to "pave the way" for bacterial superinfections, but several severe influenza cases have also been reported to develop invasive pulmonary aspergillosis [30]. An increasing amount of evidence points towards a relatively high prevalence of superinfections, including invasive aspergillosis, to be associated with COVID-19 [16-18]. However, the underlying mechanisms remain to be characterized. In the present study, we sought to understand better the biological impact of superinfections by determining and comparing the host transcriptional response to SARS-CoV-2 versus SARS-CoV-2 + Aspergillus superinfection. Collectively, our results show a much stronger host response to superinfection in the bronchial epithelial model compared to its nasal counterpart. In both models, functional analyses show that the SARS-CoV-2 + Aspergillus superinfection signature reflects important changes in the expression regulation of genes involved not only in epithelium physiology but also in the regulation of host immune and inflammatory responses compared to that of the simple SARS-CoV-2 infection.

The reconstituted HAE model of infection/superinfection appears as valuable support for the study of respiratory viral infections and virus-host interactions in highly biologically relevant experimental conditions. Previous results by our group using this model constituted of fully differentiated and functional human primary cells have provided meaningful contributions to the characterization of the kinetics of viral infection as well as on the tissue-level remodeling of the cellular ultrastructure and local innate immune responses induced by SARS-CoV-2, in line with our present observation [21]. Whereas no major differences in terms of global superinfection signatures were observed between HAE models of nasal or bronchial origin, the second part of our study highlighted more subtle differences between the two models in terms of the scale of deregulation (fold change and $p$-value), as well as in the nature of the most deregulated genes (Figure 2C). Ziegler and colleagues have recently reported that differences of infectivity and consecutive host responses between different cell subsets (type II pneumocytes, nasal goblet secretory cells) are linked to varying ACE2/TMPRSS2 levels, ACE2 expression is linked to the IFN response [31]. The discrepancies we observed in the two HAE models could be explained by differences of cell-type composition that could be interesting to explore further using combinations of additional experimental models, including ACE2/TMPRSS3 expression and single-cell RNA-seq approaches.

Our analysis of the superinfection signature revealed an important role of physiology and cilium-related genes, which could reflect an additional negative impact of Aspergillus infection on the epithelium integrity (trans-epithelial resistance, Figure 1B) and mucociliary clearance, in good agreement with previous observations by our group following different types of viral infection in HAE [19]. In the specific context of SARS-CoV-2 infection, the additional deleterious effect on epithelium integrity induced by aspergillosis might contribute to the enhanced disease severity reported in the clinic while arguably increasing the risk of additional bacterial of fungal superinfections, similarly to what has been described in the case of other respiratory viral infections [32,33].

Another major finding of our study relates to the impact of infection/superinfections on interferon and inflammatory responses, notably when compared to the limited transcriptomic data available in the literature to date. Using primary cells, Blanco-Melo and colleagues recently reported that SARS-CoV-2 induces limited IFN-I and III responses [6]. Our data are not entirely consistent with these findings. Whereas, we also demonstrate a very limited involvement of the type I interferon response in the context of simple SARS-CoV-2 infection-in contrast with data previously obtained with other viruses [19], our analysis highlights an important upregulation of crucial genes involved in the type III interferon response. This response is even more pronounced in the context of superinfection (Figure 3A,B). Type III interferon (IFN- $\lambda$ ) is known to play a key role in innate and adaptive mucosal immune responses to infection [34]. Interestingly, IFN- $\lambda$ has been identified as a critical regulator of neutrophil activation to prevent fungal infection, such as invasive pulmonary aspergillosis [35]. Our data suggest 
a divergence between type I and type III interferon responses, with enhanced activation of the latter in the context of fungal superinfection. This characteristic response could be associated with increased neutrophil activation as an important first line of adaptive defense against these two pathogens. This hypothesis is further supported by the observed induction of several monocyte-and neutrophil associated chemokines, such as CCL2, CXCL2, and CXCL3 (Figure 3A,B). Interestingly, our results suggest a strong activation of the adaptive immune response in the context of SARS-CoV-2 infection, in line with currently available clinical data from COVID-19 patients, which generally present high levels of circulating neutrophils $[1,36]$. These observations support an important role of neutrophil recruitment in response to COVID-19, more particularly in the context of fungal superinfection with an exacerbation of pro-inflammatory response. In that sense, the upregulation of key genes belonging to the IL-10 pathway observed in our analysis (Figure 2C), previously demonstrated to play a deleterious role in innate resistance to systemic aspergillosis [37], constitutes an illustration of how such "enhanced" state of inflammation could contribute to increasing severity. Our results, which give a first glimpse of the interactions between the host cell and SARS-CoV-2/Aspergillus, are to be placed in the broader and more complex context of the microbiota, which could also play a regulatory role in the severity of the pathology [38,39].

\section{Conclusions}

Our transcriptional profiling approach revealed unique features of the SARS-CoV-2 + Aspergillus superinfection signature, characterized on one side by an "enhanced" version of that induced by SARS-CoV-2 infection, but also by specific changes on respiratory tissue physiology, a distinct regulation of type I and type III interferons, and an over-induction of inflammatory response. While we acknowledge that it would be rather bold to make a statement on the possible severity associated with Aspergillus superinfection in a pre-existing COVID-19 pathological context based solely on our results, our observations suggest that the immunomodulation induced by SARS-CoV-2 infection could establish a favorable context for the development of severe forms of aspergillosis. This could constitute an important aspect to be considered for the immunological follow-up of COVID-19 patients with aspergillosis. On top of that, the characteristic infection signatures described in this study provide valuable insight in the perspective of possible future treatments targeting the COVID-19 inflammatory response, which could result in counter-productive effects for the management of aspergillosis.

Supplementary Materials: The following are available online at http://www.mdpi.com/2076-2607/8/12/1974/s1, Figure S1. Complete view of functional enrichment results for SARS-CoV2 or SARS-CoV-2+Aspergillus infected conditions vs. Mock; Figure S2. Complete view of functional enrichment results of SARS-CoV-2+Aspergillus superinfection vs. SARS-CoV2 infection; Supplementary Data File 1. Complete list of deregulated genes in SARS-CoV2 or SARS-CoV-2+Aspergillus infected conditions vs. MocK; Supplementary Data File 2. Complete list of deregulated genes in SARS-CoV-2+Aspergillus superinfection vs. SARS-CoV2 infection. Table S1. List of URLs parsed to build the databases used for enrichment analysis. Table S2. Statistics of RNA-Seq fragment pseudo-alignment to the human transcriptome.

Author Contributions: C.N.d.L., A.P., J.F., L.S., B.P., J.D., T.J., A.T., V.D., P.B., B.L., M.R.-C., and O.T. carried out the experiments and analysis of the results. C.N.L., A.P., B.L., O.T., and M.R.-C. participated to conception and coordination of the study. C.N.d.L., A.P., O.T., and M.R.-C. designed the study and wrote the manuscript. C.N.L. and A.P. contributed equally. M.R.-C. and O.T. are co-last authors. All authors have read and agreed to the published version of the manuscript.

Funding: This study was funded by Fondation Air Liquide, CNRS, and Mérieux research grants. The sponsors had no role in the study design, collection, analysis and interpretation of data, manuscript writing, or in the decision to submit the article for publication.

Acknowledgments: The authors would like to thank Epithelix (Switzerland) and IGENSEQ sequencing core facility (Institut du Cerveau ICM, Paris) for their help.

Conflicts of Interest: The authors declare no conflict of interest. The funders had no role in the design of the study; in the collection, analyses, or interpretation of data; in the writing of the manuscript; or in the decision to publish the results. 


\section{References}

1. Chen, N.; Zhou, M.; Dong, X.; Qu, J.; Gong, F.; Han, Y.; Qiu, Y.; Wang, J.; Liu, Y.; Wei, Y.; et al. Epidemiological and clinical characteristics of 99 cases of 2019 novel coronavirus pneumonia in Wuhan, China: A descriptive study. Lancet 2020, 395, 507-513. [CrossRef]

2. Guan, W.J.; Ni, Z.Y.; Hu, Y.; Liang, W.H.; Ou, C.Q.; He, J.X.; Liu, L.; Shan, H.; Lei, C.L.; Hui, D.S.C.; et al. Clinical Characteristics of Coronavirus Disease 2019 in China. N. Engl. J. Med. 2020, 382, 1708-1720. [CrossRef] [PubMed]

3. Huang, C.; Wang, Y.; Li, X.; Ren, L.; Zhao, J.; Hu, Y.; Zhang, L.; Fan, G.; Xu, J.; Gu, X.; et al. Clinical features of patients infected with 2019 novel coronavirus in Wuhan, China. Lancet 2020, 395, 497-506. [CrossRef]

4. Mehta, P.; McAuley, D.F.; Brown, M.; Sanchez, E.; Tattersall, R.S.; Manson, J.J. COVID-19: Consider cytokine storm syndromes and immunosuppression. Lancet 2020, 395, 1033-1034. [CrossRef]

5. Zhu, N.; Zhang, D.; Wang, W.; Li, X.; Yang, B.; Song, J.; Zhao, X.; Huang, B.; Shi, W.; Lu, R.; et al. A Novel Coronavirus from Patients with Pneumonia in China, 2019. N. Engl. J. Med. 2020, 382, 727-733. [CrossRef] [PubMed]

6. Blanco-Melo, D.; Nilsson-Payant, B.E.; Liu, W.-C.; Uhl, S.; Hoagland, D.; Møller, R.; Jordan, T.X.; Oishi, K.; Panis, M.; Sachs, D.; et al. Imbalanced Host Response to SARS-CoV-2 Drives Development of COVID-19. Cell 2020, 181, 1036-1045.e9. [CrossRef] [PubMed]

7. Gordon, D.E.; Jang, G.M.; Bouhaddou, M.; Xu, J.; Obernier, K.; White, K.M.; O’Meara, M.J.; Rezelj, V.V.; Guo, J.Z.; Swaney, D.L.; et al. A SARS-CoV-2 protein interaction map reveals targets for drug repurposing. Nature 2020, 583, 459-468. [CrossRef] [PubMed]

8. Messina, F.; COVID 19 INMI Network Medicine for IDs Study Group; Giombini, E.; Agrati, C.; Vairo, F.; Bartoli, T.A.; Al Moghazi, S.; Piacentini, M.; Locatelli, F.; Kobinger, G.; et al. COVID-19: Viral-host interactome analyzed by network based-approach model to study pathogenesis of SARS-CoV-2 infection. J. Transl. Med. 2020, 18, 1-10. [CrossRef] [PubMed]

9. Xiong, Y.; Liu, Y.; Cao, L.; Wang, D.; Guo, M.; Jiang, A.; Guo, D.; Hu, W.; Yang, J.; Tang, Z.; et al. Transcriptomic characteristics of bronchoalveolar lavage fluid and peripheral blood mononuclear cells in COVID-19 patients. Emerg. Microbes Infect. 2020, 9, 761-770. [CrossRef] [PubMed]

10. Zhang, Q.; Bastard, P.; Liu, Z.; Le Pen, J.; Moncada-Velez, M.; Chen, J.; Ogishi, M.; Sabli, I.K.D.; Hodeib, S.; Korol, C.; et al. Inborn errors of type I IFN immunity in patients with life-threatening COVID-19. Science 2020, 370, eabd4570. [CrossRef]

11. Bosch, A.A.T.M.; Biesbroek, G.; Trzcinski, K.; Sanders, E.A.M.; Bogaert, D. Viral and Bacterial Interactions in the Upper Respiratory Tract. PLoS Pathog. 2013, 9, e1003057. [CrossRef] [PubMed]

12. McCullers, J.A. The co-pathogenesis of influenza viruses with bacteria in the lung. Nat. Rev. Genet. 2014, 12, 252-262. [CrossRef]

13. Morens, D.M.; Taubenberger, J.K.; Fauci, A.S. Predominant Role of Bacterial Pneumonia as a Cause of Death in Pandemic Influenza: Implications for Pandemic Influenza Preparedness. J. Infect. Dis. 2008, 198, 962-970. [CrossRef] [PubMed]

14. Paget, C.; Trottein, F. Mechanisms of Bacterial Superinfection Post-influenza: A Role for Unconventional T Cells. Front. Immunol. 2019, 10, 336. [CrossRef]

15. Bengoechea, J.A.; Bamford, C.G. SARS-CoV-2, bacterial co-infections, and AMR: The deadly trio in COVID-19? EMBO Mol. Med. 2020, 12. [CrossRef]

16. Zhou, F.; Yu, T.; Du, R.; Fan, G.; Liu, Y.; Liu, Z.; Xiang, J.; Wang, Y.; Song, B.; Gu, X.; et al. Clinical course and risk factors for mortality of adult inpatients with COVID-19 in Wuhan, China: A retrospective cohort study. Lancet 2020, 395, 1054-1062. [CrossRef]

17. Lescure, F.-X.; Bouadma, L.; Nguyen, D.; Parisey, M.; Wicky, P.-H.; Behillil, S.; Gaymard, A.; Bouscambert-Duchamp, M.; Donati, F.; Le Hingrat, Q.; et al. Clinical and virological data of the first cases of COVID-19 in Europe: A case series. Lancet Infect. Dis. 2020, 20, 697-706. [CrossRef]

18. Alanio, A.; Dellière, S.; Fodil, S.; Bretagne, S.; Mégarbane, B. Prevalence of putative invasive pulmonary aspergillosis in critically ill patients with COVID-19. Lancet Respir. Med. 2020, 8, e48-e49. [CrossRef] 
19. De Lamballerie, C.N.; Pizzorno, A.; Dubois, J.; Julien, T.; Padey, B.; Bouveret, M.; Traversier, A.; Legras-Lachuer, C.; Lina, B.; Boivin, G.; et al. Characterization of cellular transcriptomic signatures induced by different respiratory viruses in human reconstituted airway epithelia. Sci. Rep. 2019, 9, 1-12. [CrossRef]

20. Pizzorno, A.; Terrier, O.; De Lamballerie, C.N.; Julien, T.; Padey, B.; Traversier, A.; Roche, M.; Hamelin, M.-E.; Rhéaume, C.; Croze, S.; et al. Repurposing of Drugs as Novel Influenza Inhibitors From Clinical Gene Expression Infection Signatures. Front. Immunol. 2019, 10, 60. [CrossRef]

21. Pizzorno, A.; Padey, B.; Julien, T.; Trouillet-Assant, S.; Traversier, A.; Errazuriz-Cerda, E.; Fouret, J.; Dubois, J.; Gaymard, A.; Lescure, F.-X.; et al. Characterization and Treatment of SARS-CoV-2 in Nasal and Bronchial Human Airway Epithelia. Cell Rep. Med. 2020, 1, 100059. [CrossRef] [PubMed]

22. Martin, M. Cutadapt removes adapter sequences from high-throughput sequencing reads. EMBnet J. 2011, 17, 10-12. [CrossRef]

23. Bray, N.L.; Pimentel, H.; Melsted, P.; Pachter, L. Near-optimal probabilistic RNA-seq quantification. Nat. Biotechnol. 2016, 34, 525-527. [CrossRef] [PubMed]

24. Maglott, D.R.; Ostell, J.; Pruitt, K.D.; Tatusova, T. Entrez Gene: Gene-centered information at NCBI. Nucleic Acids Res. 2004, 33, D54-D58. [CrossRef] [PubMed]

25. Soneson, C.I.; Love, M.; Robinson, M.D. Differential analyses for RNA-seq: Transcript-level estimates improve gene-level inferences. F1000Research 2016, 4, 1521. [CrossRef]

26. Love, M.I.; Huber, W.; Anders, S. Moderated estimation of fold change and dispersion for RNA-seq data with DESeq2. Genome Biol. 2014, 15, 550. [CrossRef]

27. Breuza, L.; Poux, S.; Estreicher, A.; Famiglietti, M.L.; Magrane, M.; Tognolli, M.; Bridge, A.; Baratin, D.; Redaschi, N. The UniProt Consortium The UniProtKB guide to the human proteome. Database 2016, 2016. [CrossRef]

28. Grossmann, S.; Bauer, S.; Robinson, P.N.; Vingron, M. Improved detection of overrepresentation of Gene-Ontology annotations with parent child analysis. Bioinformatics 2007, 23, 3024-3031. [CrossRef]

29. Clancy, C.J.; Nguyen, M.H. Coronavirus Disease 2019, Superinfections, and Antimicrobial Development: What Can We Expect? Clin. Infect. Dis. 2020. [CrossRef]

30. Verweij, P.E.; Gangneux, J.-P.; Bassetti, M.; Brüggemann, R.J.M.A.; Cornely, O.; Koehler, P.; Lass-Flörl, C.; Van De Veerdonk, F.L.; Chakrabarti, A.; Hoenigl, M. Diagnosing COVID-19-associated pulmonary aspergillosis. Lancet Microbe 2020, 1, e53-e55. [CrossRef]

31. Ziegler, C.G.; Allon, S.J.; Nyquist, S.K.; Mbano, I.M.; Miao, V.N.; Tzouanas, C.N.; Cao, Y.; Yousif, A.S.; Bals, J.; Hauser, B.M.; et al. SARS-CoV-2 Receptor ACE2 Is an Interferon-Stimulated Gene in Human Airway Epithelial Cells and Is Detected in Specific Cell Subsets across Tissues. Cell 2020, 181, 1016-1035.e19. [CrossRef] [PubMed]

32. Smith, C.; Kulkarni, H.; Robert, H.; Williams, G.; Andrew, P.; Easton, A.; O'Callaghan, C. Influenza virus infection of human ciliated respiratory epithelial cells in culture. Eur. Respir. J. 2013, 42 (Suppl. S57), 4406.

33. Wu, N.-H.; Yang, W.; Beineke, A.; Dijkman, R.; Matrosovich, M.; Baumgärtner, W.; Thiel, V.E.; Valentin-Weigand, P.; Meng, F.; Herrler, G. The differentiated airway epithelium infected by influenza viruses maintains the barrier function despite a dramatic loss of ciliated cells. Sci. Rep. 2016, 6, 39668. [CrossRef] [PubMed]

34. Ye, L.; Schnepf, D.; Staeheli, P. Interferon- $\lambda$ orchestrates innate and adaptive mucosal immune responses. Nat. Rev. Immunol. 2019, 19, 614-625. [CrossRef] [PubMed]

35. Espinosa, V.; Dutta, O.; Mc Elrath, C.; Du, P.; Chang, Y.-J.; Cicciarelli, B.; Pitler, A.; Whitehead, I.; Obar, J.J.; Durbin, J.E.; et al. Type III interferon is a critical regulator of innate antifungal immunity. Sci. Immunol. 2017, 2, eaan5357. [CrossRef]

36. Qin, C.; Zhou, L.; Hu, Z.; Zhang, S.; Yang, S.; Tao, Y.; Xie, C.; Ma, K.; Shang, K.; Wang, W.; et al. Dysregulation of Immune Response in Patients With Coronavirus 2019 (COVID-19) in Wuhan, China. Clin. Infect. Dis. 2020, 71, 762-768. [CrossRef]

37. Clemons, K.V.; Grunig, G.; Sobel, R.A.; Mirels, L.F.; Rennick, D.M.; Stevens, D.A. Role of IL-10 in invasive aspergillosis: Increased resistance of IL-10 gene knockout mice to lethal systemic aspergillosis. Clin. Exp. Immunol. 2000, 122, 186-191. [CrossRef]

38. Santacroce, L.; Charitos, I.A.; Ballini, A.; Inchingolo, F.; Luperto, P.; De Nitto, E.; Topi, S. The Human Respiratory System and its Microbiome at a Glimpse. Biology 2020, 9, 318. [CrossRef] 
39. Bao, L.; Zhang, C.; Dong, J.; Zhao, L.; Li, Y.; Sun, J. Oral Microbiome and SARS-CoV-2: Beware of Lung Co-infection. Front. Microbiol. 2020, 11, 1840. [CrossRef]

Publisher's Note: MDPI stays neutral with regard to jurisdictional claims in published maps and institutional affiliations.

(C) 2020 by the authors. Licensee MDPI, Basel, Switzerland. This article is an open access article distributed under the terms and conditions of the Creative Commons Attribution (CC BY) license (http://creativecommons.org/licenses/by/4.0/). 